\title{
FUZZY MODELLING OF POWER SYSTEM OPTIMAL LOAD FLOW
}

V. Miranda
J. T. Saraiva

FEUP - DEEC - Faculdade de Engenharia da Universidade do Porto

INESC - Instituto de Engenharia de Sistemas e Computadores

$\mathrm{Lg}^{\circ}$ de Mompilher - 4000 PORTO - PORTUGAL

Tel: 351-2-321006 FAX: 351-2-318692 Ean: v_miranda@inescn.ctt.pt

\begin{abstract}
In this paper, a fuzzy model for power system operation is presented. Uncertainties in loads and generations are modelled as fuzzy numbers. System behavior under known (while uncertain) injections is dealt with by a DC fuzzy power flow model. System optimal (while uncertain) operation is calculated with linear programming procedures where the problem nature and structure allows some efficient techniques such as Dantzig Wolfe decomposition and dual simplex to be used. Among the results, one obtains a fuzzy cost value for system operation and possibility distributions for branch power flows and power generations. Some risk analysis is possible, as system robustness and exposure indices can be derived and hedging policies can be investigated.
\end{abstract}

\section{INTRODUCTION}

Recently, some papers have been published on the subject of "fuzzy load flow" [1,2]. Uncertainty in loads and power generations was directly taken in account with the help of concepts from the fuzzy set theory. In fact, one of Lofti Zadeh's contributions to system modelling is the representation of vague, incomplete knowledge or qualitative information that does not have a random nature and therefore cannot be represented by a probabilistic approach. Fuzzy set theory provided also a basis for the interpretation of membership functions as possibility distributions $[3,4,5,6]$, which is a very useful concept in many practical applications.

Fuzzy load data are given under the form of possibility distributions for loads and generations. These distributions can be derived from qualitative assessment or linguistic declarations such as a simple " load in bus k will be more or less 10 MW". In this paper, we will not go deeply into these representation techniques, but several approaches have been proposed and we would like to mention references $[7,8]$ for their simplicity and psychological background. In planning studies, uncertainty in loads derives from load forecasting which is, in one way or another, related to the economic and political scenarios that have been considered.
The results of a fuzzy load flow study are mainly the possibility distributions of line power flows and node voltages. Some immediate and interesting conclusions may be obtained from them, considering that they represent globally and in a lumped form a whole set of scenarios examined at one time, with their degree of possibility (or credibility, if desired). Line flow and node voltage sensitivity to power injection uncertainty are readily available, and "what may happen" in a human sense can be provided as a result, with increased information value if channeled through an adequate man-machine interface.

As fuzzy flows are related to a feasibility idea, one would like to go a little further into an operation concept, in the sense that power generation is driven by economics and therefore uncertainty in future loads implies uncertainty in dispatch decisions. The present paper presents a first approach to this concern.

In order to represent this operational feature, one must in some way try to "optimize" the uncertainty in generation costs. As this means combining optimization and power flows, we reach a fuzzy optimal power flow (FOPF) workframe; at the present stage of evolution, the tool developed uses DC fuzzy load flow model and linear programming.

As it will be shown, FOPF can be used not only to calculate power injection and line flow possibility distributions (under the assumption that the system will be operated as economically as possible) but also to assess the robustness of the system performance and the degree of exposure to an uncertain future.

\section{ABOUT FUZZY SET THEORY}

\section{Basic concepts}

Fuzzy Set theory can be considered as an extension of $n$-valued logic if the number of the admissible logic values tends to infinity. For instance, given a subset $X_{1}$ of an universe $X$, the membership degree of an element $X_{1}$ to $X_{1}$ is included in:

$-\{0,1\}$ in Boole's logic;

- $\{0,0.5,1\}$ in Lukasiewicz's trivalued logic;

- $[0,1]$ if normalized fuzzy sets are considered.

According to (1), a fuzzy set $\tilde{A}$ is characterized by a membership function $\mu \tilde{A}(x)$ relating each element $\mathrm{x}_{1}$ to its compatibility degree with $\mathrm{X}_{1}$. In this case, the transition between the extreme situations of full and 
complete lack of membership is gradual. In certain circumstances, a fuzzy set can be associated to a possibility distribution. In such cases, the membership function corresponds to a possibility distribution function.

$$
\tilde{A}=\left\{\left(x_{1}, \mu \tilde{A}\left(x_{1}\right)\right), x_{1} \in X_{1}\right\}
$$

An $\alpha$-level set or an $\alpha$-core of a fuzzy set $\tilde{A}$ defined in $X_{1}$ is the hard set $A_{\alpha}$ obtained from $\tilde{A}$ for each $\alpha \in[0,1]$ such that:

$$
A_{\alpha}=\left\{x_{1} \in X_{1}: \mu \tilde{A}\left(x_{1}\right) \geq \alpha\right\}
$$

The support set of a fuzzy set $\tilde{A}, S(\tilde{A})$, is the hard set containing the elements of $X_{1}$ having their membership function in $] 0,1]$.

A fuzzy set $\tilde{A}$ is said to be a fuzzy number, $F N$, if it is a convex fuzzy set of the real line $R$ such that its membership function is piecewise continuous.

\section{Algebraic operations involving fuzzy sets}

The usual algebraic operations can be readily extended to fuzzy sets using the Extension Principle formulated by Zadeh. In particular, given the fuzzy sets $\tilde{A}$ and $\tilde{O}$, the addition $\tilde{A}+\tilde{O}$ is calculated by (4) and (5). The product of a fuzzy set $\tilde{A}$ by a real $c$ is given by (6) and (7).

$$
\begin{aligned}
& \tilde{O}=\left\{\left(\mathrm{y}_{1}, \mu \tilde{O}\left(\mathrm{y}_{1}\right)\right), \mathrm{y}_{1} \in \mathrm{X}_{1}\right\} \\
& \begin{array}{r}
\tilde{\mathrm{A}}+\tilde{O}=\left\{\left(\mathrm{z}_{1}, \mu \tilde{\mathrm{A}}+\tilde{O}\left(\mathrm{z}_{1}\right)\right), \mathrm{z}_{1} \in \mathrm{X}_{1}\right\} \\
\mu \tilde{A}+\tilde{O}\left(\mathrm{z}_{1}\right)=\max \left\{\min \left[\mu \tilde{A}\left(\mathrm{x}_{1}\right), \mu \tilde{O}\left(\mathrm{y}_{1}\right)\right]\right\} \\
\mathrm{z}_{1}=\mathrm{x}_{1}+\mathrm{y}_{1}
\end{array} \\
& \text { c. } \tilde{A}=\left\{\left(\mathrm{x}_{1}, \mu_{\mathrm{C}} \tilde{A}\left(\mathrm{x}_{1}\right)\right), \mathrm{x}_{1} \in \mathrm{X}_{1}\right\} \\
& \mu_{\mathrm{C}} \tilde{\mathrm{A}}(\mathrm{x})=\mu \tilde{A}(\mathrm{c} . \mathrm{x})
\end{aligned}
$$

It should be emphasized that the max-min convolution used to extend several algebraic operations fuzzy sets is much simpler than if probabilistic concepts are considered. This fact strongly contributes to make algebraic operations with fuzzy sets very efficient in a computational framework.

\section{Ordering (?) of fuzzy numbers [9]}

The removal of a fuzzy set $\tilde{A}$ regarding a real $\mathrm{k}, \operatorname{Rem}(\tilde{\mathrm{A}}, \mathrm{k})$, is defined as the mean value of its left and right removals which correspond to the areas $A_{1}$ and $A_{1}+A_{2}$ of Fig. 1, respectively.

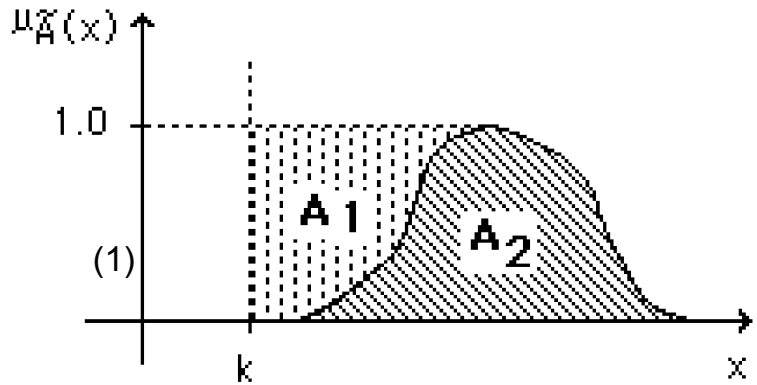

Fig. 1. Left and right removals of a fuzzy number $\tilde{A}$.

$\operatorname{Rem}(\tilde{\mathrm{A}}, \mathrm{k})$ can be considered to be associated to the distance from $\tilde{A}$ to $k$. However, this ordering is not strict. In other words, in general there is not an injective correspondence between a set of fuzzy sets and $\mathrm{R}$. In the attempt to establish an ordering, some undecided situations may be solved with other criteria such as the mean value or the amplitude of $S(\tilde{A})$. For practical purposes, however, the successive application of these three criteria is satisfactory.

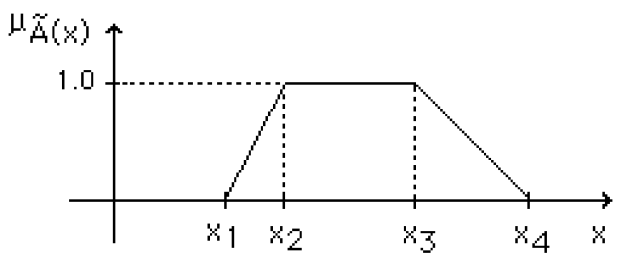

Fig. 2. Trapezoidal fuzzy number.

If one considers the trapezoidal $F N \tilde{A}$ represented in Fig. 2, its removal for $k=0$ is given by

(3)

$$
\operatorname{Rem}(\tilde{\mathrm{A}}, 0)=\left(\mathrm{x}_{1}+\mathrm{x}_{2}+\mathrm{x}_{3}+\mathrm{x}_{4}\right) / 4
$$

${ }^{4} \mathrm{~A}$ FN such as $\tilde{A}$ is usually represented by its breaking points:

$$
\tilde{A}<=>\left(x_{1}, x_{2}, x_{3}, x_{4}\right)
$$

\section{(GNCERTAIN LOAD AND GENERATION REPRESENTATION}

(7)

An uncertain load will be represented by a fuzzy number which is in fact a membership function over the real set. FNs may have a variety of shapes (within some conditions) but for simplicity we will deal mainly with data represented by trapezoidal membership functions (with triangular ones as a special case). A linguistic declaration such as "peak values will surely not occur below 10 or above $30 \mathrm{MW}$, and the best estimate is, say, between 20 and $25 \mathrm{MW}^{\prime \prime}$

will be translated into a FN such as in Fig. 3.

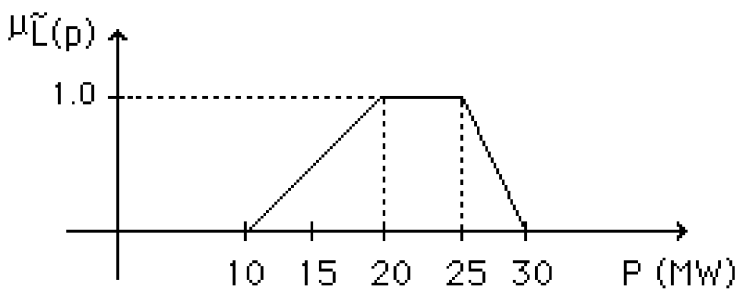


Fig. 3 - FN representing a qualitative linguistic load prediction.

As far as generation is concerned, one must distinguish two cases, namely controllable and uncontrolable power sources:

a) controllable power sources - they refer to power stations or other power injection facilities operated by the utility who performs the study: their possibility distributions will come as a result of the FOPF study;

b) uncontrolable power sources - in some studies, these are related with independent power producers that due to governmental or contract regulations can inject power in the system without dispatch control; in other cases (mainly in distribution systems), these may represent the presence of eolic sources: their possibility distributions must be given as data to the model.

\section{PROPOSED METHODOLOGY}

The OPF is usually defined as an optimization problem where one aims at identifying the best operation point of an electrical network under some criteria subject to operational and security constraints [10].

When loads or generations are described in terms of possibility distributions the problem turns into Fuzzy Optimal Power Flow. In this paper, we use a DC fuzzy power flow model, for its simplicity. In the following paragraphs, a general incremental approach is adopted so that linearization errors and problem dimension are kept within acceptable limits.

\section{Fuzzy DC Power Flow}

A fuzzy description of the bus angles and active power flows can be obtained using an incremental DC model considering the following steps:

a) a deterministic DC load flow is previously run using the specified injected active powers associated with the medium point of their possibility distributions. Thus, deterministic values for the angles $\left(\theta_{d}\right)$ and active power flows $\left(\mathrm{P}_{\mathrm{dik}}\right)$ are obtained.

b) the possibility distributions $\left(\Delta \mathrm{P}_{\mathrm{i}}\right)$ of the deviations from the specified active injected powers $\left(P_{i}\right)$ regarding the deterministic values $\left(P_{\text {di }}\right)$ are evaluated.

c) the possibility distributions of both bus angle deviations and active power flow deviations can be evaluated considering the DC model matrix [B] and the sensitivity coefficient matrix $[A]$. The possibility distributions of bus angles and active power flows are obtained by superimposing their respective increments to the deterministic values.

$$
\begin{aligned}
& {[\Delta \theta]=[\mathrm{B}]^{-1} \cdot[\Delta \mathrm{P}]} \\
& {\left[\Delta \mathrm{P}_{\mathrm{ik}}\right]=[\mathrm{A}] \cdot[\Delta \mathrm{P}]}
\end{aligned}
$$

\section{Generator modelling}

The generator cost functions are linearized in a known way namely for the generators having non linear convex cost functions. These are decomposed in two or more linear sections according to its nonlinearity degree. In other words, these generators are represented by a number of fictitious generators having constant incremental costs.

\section{Objective function}

The attribute to be minimized is the (uncertain) cost of energy generation needed to meet uncertain load requirements. It is translated into an objective function resulting from the addition of fuzzy numbers. The ordering in the attribute space is imposed by taking the removal of the fuzzy objective value.

$$
\underset{i=1}{\mathrm{Z}=\Sigma a_{i} \cdot G_{i}}
$$

where

- ng- number of generators;

- $a_{i}$ - incremental cost of generator $i$;

- $G_{i}$ - fuzzy generation at bus $i$.

\section{Operational constraints}

As we use a DC power flow formulation, no transmission losses are considered. Therefore, the uncertainty in loads is directly related to uncertainty in generations. If loads and generations are represented by trapezoidal FNs, one has the following power balance equation

$$
\begin{aligned}
& \stackrel{\sum}{n g} G_{i}+G_{u c}=L \\
& \quad G i<=>\left(G_{i 1}, G_{i 2}, G_{i 3}, G_{i 4}\right) \\
& \quad G_{u c}<=>\left(G_{u c 1}, G_{u c 2}, G_{u c 3}, G_{u c 4}\right) \\
& \quad L \ll=>\left(L_{1}, L_{2}, L_{3}, L_{4}\right)
\end{aligned}
$$

where generator

- $G_{i}$ - generation possibility distribution of

- $G_{u c}$ - possibility distribution of generated power by uncontrolled sources;

- L - total load possibility distribution;

The deconvolution of this fuzzy equation can be achieved by decomposing it in the following four algebraic equations:

$$
\underset{i=1}{n g} G_{i k}=L_{k}-G_{u c k} \quad k=1, \ldots, 4
$$

Security constraints 
The security constraints are considered in classical OPF studies to obtain operating points satisfying certain maximum and minimum values of some variables. In the FOPF model, constraints related to thermal limits in the network branches are included under the form of power flow limits. The constraint corresponding to branch I is given by:

$$
] \mathrm{P}_{\operatorname{lmin}}, \mathrm{P}_{\mathrm{Imax}}\left[\supset \mathrm{S}\left(\mathrm{P}_{\mathrm{l}}\right) \quad \mathrm{l}=1, \ldots, \mathrm{nl}\right.
$$

where power flow;

- $P_{I}$ - possibility distribution of line I active

- $\mathrm{P}_{\text {Imin }}$ and $\mathrm{P}_{\text {Imax }}$ - extreme admissible values of branch I active power flow;

- $\mathrm{nl}$ - number of network branches.

It should be stressed that using the known sensitivity coefficient matrix $[A]$, it is possible to express this type of constraints in terms of the injected powers and, thus, of the control variables.

\section{Complete mode}

The complete model is a minimization problem formed by objective function (12) and constraints (17) and (18). Besides, the following constraints related to generators must also be included:

$$
\mathrm{G}_{\mathrm{imin}} \leq \mathrm{G}_{\mathrm{i} 1} \leq \mathrm{G}_{\mathrm{i} 2} \leq \mathrm{G}_{\mathrm{i} 3} \leq \mathrm{G}_{\mathrm{i} 4} \leq \mathrm{G}_{\mathrm{imax}} \quad \mathrm{i}=1, \ldots, \mathrm{ng}
$$

These constraints are included to ensure that generation possibility distributions preserve the increasing nature of their support sets.

Considering a network having ng generators and $\mathrm{nl}$ branches this model incorporates:

- 4.ng variables;

$-4+5 . n g+5 . n l$ constraints.

The high number of constraints and variables not only suggests but also advises the use of an incremental methodology when a FOPF study is to be performed considering a large network.

\section{FOPF algorithm}

The proposed incremental methodology starts from an operating point that satisfies the power balance equation. The violated limits of generation and power flows are then identified. After selecting the most severe power flow violation the related constraints are incorporated in an incremental optimization model. As a result of this incremental study, one gets the deviations of the initial generations needed to cancel the selected violation. The number of violated limits of both generation and power flows is generally a small percentage of the total possible ones. Thus, the optimal solution can be identified by solving problems of small dimension when compared to the complete ones. The FOPF algorithm flowchart is presented in Fig. 4. Its basic steps will be described in the following paragraphs.

A - Considering that loads and generations are represented by trapezoidal fuzzy numbers, the basic fuzzy dispatch is formulated as:

$$
\begin{aligned}
& \operatorname{Min} 718)_{i=1}^{n g} a_{i} . \sum_{k=1}^{4} G_{i k} / 4 \\
& n g \\
& \sum_{i=1}^{n} G_{i k}=L_{k}-G_{u c k} \quad k=1, \ldots, 4 \\
& G_{i \min } \leq G_{i 1} \leq G_{i 2} \leq G_{i 3} \leq G_{i 4} \leq G_{i \max } i=1, \ldots, n g
\end{aligned}
$$

This is a linear optimization problem having four coupling constraints and ng sets of constraints each one related to each generator. The coefficient matrix of this problem has a block diagonal structure so that the Dantzig Wolfe decomposition principle can be used to solve it. In this case, the master problem has $4+n g$ constraints and there are ng subproblems having, each one, 5 constraints.

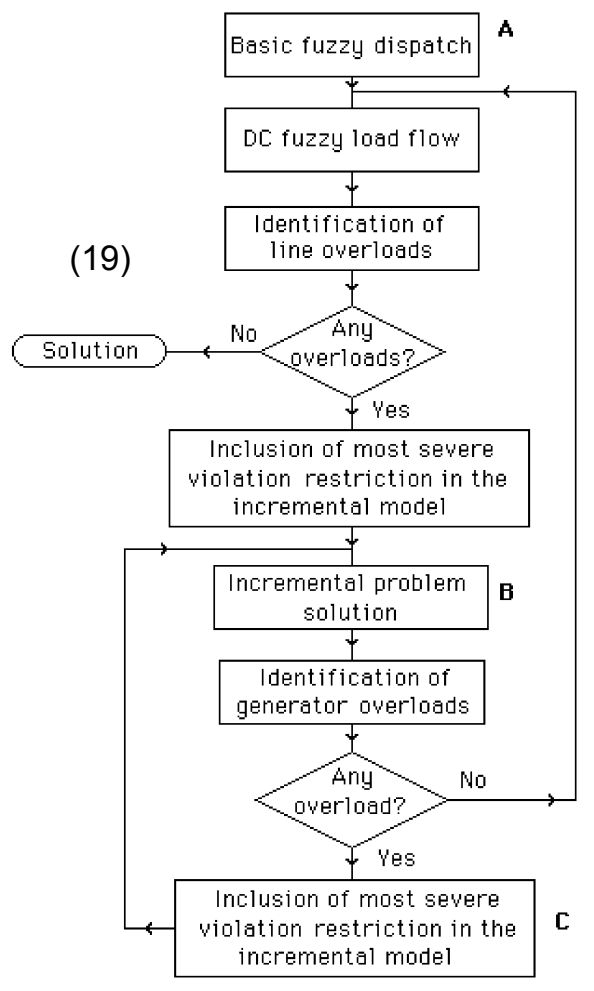

Fig. 4. FOPF algorithm flowchart.

B - The linear incremental model is used to redispatch some generations so that the most severe power flow violation is canceled. Let us consider deviations $\Delta \mathrm{G}_{\mathrm{ik}}$ $(k=1, \ldots, 4)$ related to the several generators. These variables can have either negative or positive values so that they will have to be substituted by differences of non-negative ones. The linear incremental model is formed by: 
a) Power balance incremental equation

If the initial basic generations are to be changed by $\Delta G_{i k} \quad(k=1, \ldots, 4)$, the power balance incremental equation is given by:

$$
\underset{\mathrm{i}=1}{\mathrm{ng}} \Delta \mathrm{G}_{\mathrm{ik}}=0 \quad \mathrm{k}=1, \ldots, 4
$$

b) Incremental constraints related to the most severe power flow violation

Let us suppose the violation of the maximum power flow limit on branch I was found to be the most severe one. This power flow can be expressed, through sensitivity coefficients, in terms of the injected powers. As it is only possible to change the generated powers of controlled generators one would get:

$$
\begin{aligned}
& \text { ng } \mathrm{n} \\
& \left(\mathrm{P}_{\mathrm{l}}+\Delta \mathrm{P}_{\mathrm{l}}\right)=\Sigma \mathrm{a}_{\mathrm{li}} \cdot\left(\mathrm{P}_{\mathrm{i}}+\Delta \mathrm{P}_{\mathrm{i}}\right)+\sum \mathrm{a}_{\mathrm{li}} \cdot \mathrm{P}_{\mathrm{i}} \\
& \mathrm{i}=2 \quad \mathrm{i}=\mathrm{ng}+1
\end{aligned}
$$

where

- $\left(P_{1}+\Delta P_{1}\right)$ - new possibility distribution of branch I power flow;

- $\left(\mathrm{P}_{\mathrm{i}}+\Delta \mathrm{P}_{\mathrm{i}}\right)$ - new possibility distribution of controlled i node injected power;

- $P_{i}$ - possibility distribution of injected power other nodes;

- $a_{\text {|i }}$ - sensitivity coefficient of branch $1 /$ node $\mathrm{i}$;

- $\mathrm{n}$ - number of network nodes. Node 1 is assumed to be the angle reference.

As loads possibility distributions are constant, the deviations of the ng injected powers are, in fact, deviations of controlled generations. Thus, it is possible to derive the following four constraints:

$$
\begin{aligned}
& \Sigma a_{\text {li }} \Delta G_{i k}+\Sigma a_{\mid i} \cdot \Delta G_{i(5-k)} \leq P_{\text {Imax }}-P_{\mid k} \quad k=1, \ldots, 4 \\
& i \in n g 1 \quad i \in n g 2
\end{aligned}
$$

where

- ng1 and ng2 - subsets of nodes having controlled generators whose elements in branch I of the sensitivity coefficient matrix $[A]$ are either positive or negative;

- PImax - branch I power flow maximum admissible value;

- $P_{l k}$ - $k$ element of branch I power flow possibility distribution.

\section{c) Constraints related to the generators}

The incremental model will also have to incorporate constraints to ensure that the increasing nature of the generation possibility distribution support set is not violated. In incremental terms we get:
$\mathrm{G}_{i k}+\Delta \mathrm{G}_{\mathrm{ik}} \leq \mathrm{G}_{\mathrm{i}(\mathrm{k}+1)}+\Delta \mathrm{G}_{\mathrm{i}(\mathrm{k}+1)} \mathrm{k}=1,2,3 \mathrm{i}=1, \ldots, \mathrm{ng}$

\section{d) Objective function}

The objective function to be minimized is now given by the summation of the production costs related to the initial possibility distribution deviations. Thus, we have:

$$
\Delta Z=\sum_{\mathrm{i}=1}^{\mathrm{ng}} \underset{\mathrm{k}=1}{\operatorname{ar}} \stackrel{\sum}{\sum} \Delta \mathrm{G}_{\mathrm{ik}} / 4
$$

The coefficient matrix of this linear problem keeps a block diagonal structure and the Dantzig Wolfe decomposition principle can be still applied.

C - The generation possibility distributions can now be recalculated as a result of solving the previous incremental problem. Regarding generator $\mathrm{i}$ one would get:

$G_{i}{ }^{\prime}<=>\left(G_{i 1}+\Delta G_{i 1}, G_{i 2}+\Delta G_{i 2}, G_{i 3}+\Delta G_{i 3}, G_{i 4}+\Delta G_{i 4}\right)(28)$

These new distributions can violate either their minimum or maximum admissible values. If the minimum was violated, constraint (29) will be incorporated in the incremental model, while this will occur with constraint (30) if the maximum was violated.

$$
\begin{aligned}
& G_{i 1}+\Delta G_{i 1} \leq G_{i \min } \\
& G_{i 4}+\Delta G_{i 4} \leq G_{i \max }
\end{aligned}
$$

The block diagonal structure of the coefficient matrix is not destroyed by the successive incorporation of these constraints. The solution of the augmented problem from the beginning is not necessary if one considers that the solution of the incremental problem obtained in the previous iteration remains optimal but not admissible. This fact suggests the use of dual simplex techniques to overcome the non admissibility.

\section{Solution using $\alpha$-cuts}

In the previous iteration scheme it was considered that generator and power flow possibility distributions were always described by trapezoidal fuzzy numbers. However, when a violation, as represented in Fig. 5, occurs the problem can be solved by redispatching only the fuzzy subset between $\mathrm{P}_{\mathrm{Imax}}$ and $\mathrm{P}_{14}$. This can be achieved considering, in the generator possibility distributions, variables related to this $\alpha$-cut.

This kind of situation can also occur when a generator limit violation is identified. This technique leads to a new way of looking at the objective function as the removal expression changes when a new $\alpha$-cut is considered. The expression of the objective function must be changed to the new expression of 
the removal, but nevertheless it remains linear. Besides, a progressive increasing

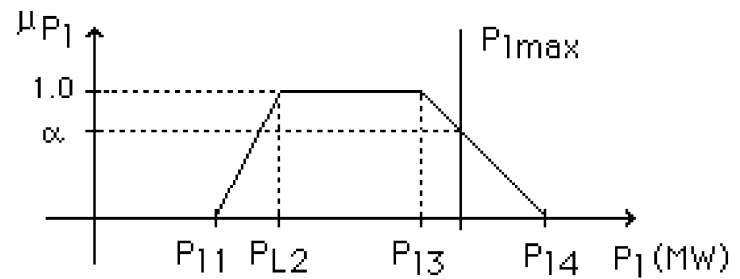

Fig. 5. Violation of a power flow related to an $\alpha$ - cut.

of problem dimension occurs both in terms of variables and constraints. However, the generation and power flow possibility distributions will also be allowed to lose their initial trapezoidal shape. Thus, one gets a more flexible problem as there is a larger number of possible solutions to be analyzed. Finally, it should be emphasized that this problem remains linear as the generator possibility distributions are characterized by linear sections between $\alpha$-cuts.

\section{ROBUSTNESS, EXPOSURE AND HEDGING}

The concepts of robustness and exposure are part of the general workframe of risk analysis; on power system applications they have been well summarized in ref. [11].

If, as a result of a FOPF study, a feasible solution for the possibility distributions of both power injection and line power flow has been reached, then the configuration under study must be taken as fully robust for the whole set of scenarios underlying the assumed load uncertainties.

However, many cases will only be possible for a given $\alpha$-cut level or above. Below that level, either line power flow possibility at some lines or power generation possibility at some nodes go beyond preset limits. In this case, the system may be said as (1- $\alpha$ ) robust, having an $\alpha$ degree of exposure. This degree comes associated with the network element which has its limit violated for a higher $\alpha$ value. One may therefore rank system components as for exposure degrees by systematically allowing the relaxation of the most exposed constraint (by, for example, a reasonable power step) and so identify the critical elements in a network for which reinforcement strategies should first be considered.

For instance, if one finds that a link $\mathrm{k}$ imposes a degree of exposure of 0.6 and reinforcing its capacity (say by an amount of $10 \mathrm{MW}$ ) causes another line $\mathrm{j}$ to impose a degree of exposure of 0.4 , one can say that an exposure reduction of 0.2 could be obtained at the cost of increasing $10 \mathrm{MW}$ in line $\mathrm{k}$ capacity. We are therefore also in face of a proposal for a hedging policy.

It must be underlined that the measure of degree of exposure mentioned above is not at all related with probabilistic concepts, and thus has not been expressed in percentage values. It depends only on the qualitative assessment by the engineer or the decision maker of the possibility of different future scenarios (or their degree of credibility). In this we differ from other proposals that deal with this same problem, where a confusion between probabilities (subjective probabilities, in fact) and possibilities may be detected. Usually these items are not dealt within a rigorous theoretical frame.

It must also be said that an approach by bounded intervals is coherent and may be seen as a particular case of a fuzzy set approach, where for instance the membership functions would be all rectangular and with value 1 over the intervals and 0 outside.

\section{Indices of robustness and exposure calculation}

The indices of robustness and exposure of an electric system can be evaluated by performing several incremental studies for different $\alpha$-cut levels. The selection of the next $\alpha$-cut to be used can be guided by a process similar to the bisection method applied, for instance, to the solution of non linear equations. Admit that it is found that the system has no solution for the initial specified imprecision, that is for $\alpha=0$; in the next step the $\alpha=1$ level will be tried. If the FOPF doesn't also have a solution for this level the system can't even accommodate the loads related to the least specified imprecision. If there is a solution for $\alpha=1$ another incremental study for $\alpha=0.5$ will be run. This iterative process can go on until a desired pre-established precision is obtained.

\section{Improvement of system robustness}

When one wishes to improve the system robustness index, two questions can be devised:

a) How will it be possible to improve the system robustness index by a given amount?

To answer this question it is essential to identify the constraint, or constraints, whose violation is responsible for a given value of the robustness index. This can be achieved, for instance, by performing an incremental study for a level just below the exposure index $\alpha$. This study won't have a solution but it will be possible to identify the referred constraints through the inspection of the simplex tableau available when the non admissibility is detected. The resources related to those constraints will be the ones to be increased or decreased, depending on the constraints, if the robustness index is to be improved. The quantification of the resources to be altered will be achieved by performing a number of incremental FOPF studies for increased or decreased values of those resources until the desired $\alpha$ is reached. In fact, this corresponds to a parametric 
study involving the independent terms of some constraints of the linear incremental model.

b) What will it be the impact on $\alpha$ of increasing a generator or power flow limit?

Let us suppose that a constraint responsible for a given level of the exposure index is identified. The planner admits a change in the related resource by a given amount. However, he wishes to estimate the improvement of the exposure index. This evaluation can be obtained, for instance, by performing an iterative bisection process similar to the one previously described. The levels to be investigated are now in the interval $[0, \alpha[$, where $\alpha$ is the initial exposure index.

\section{APPLICATION EXAMPLE}

\section{System data}

For the sake of clarity, the proposed methodology is exemplified using a very small system such as the 6 bus/ 6 branch network presented in Fig. 6 . The corresponding data are given in tables 1,2 and 3.

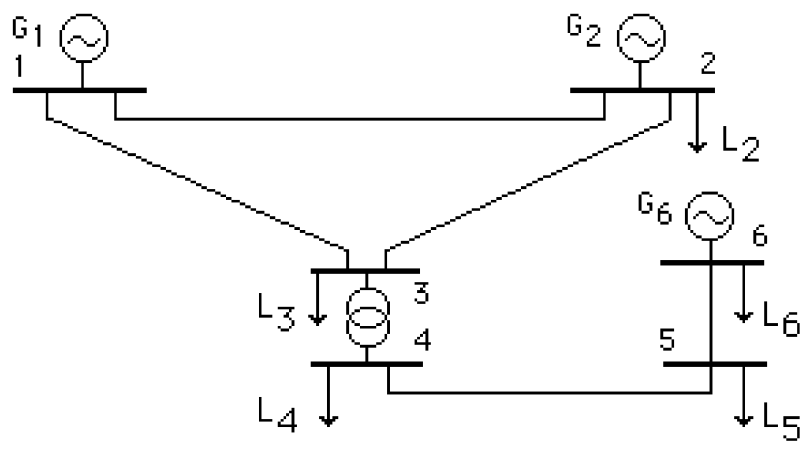

Fig. 6. Network used to exemplify the proposed methodology.

\begin{tabular}{|ccccc|}
\hline generator & section & $\begin{array}{c}\text { Gimim } \\
(\mathrm{MW})\end{array}$ & $\begin{array}{c}\text { Gimax } \\
(\mathrm{MW})\end{array}$ & $\begin{array}{c}\text { increm. cost } \\
(\$ / \text { MWhour })\end{array}$ \\
1 & 1 & 0.0 & 50.0 & 1000.0 \\
1 & 2 & 0.0 & 40.0 & 3000.0 \\
1 & 3 & 0.0 & 40.0 & 6000.0 \\
2 & 1 & 20.0 & 60.0 & 500.0 \\
2 & 2 & 0.0 & 50.0 & 1000.0 \\
2 & 3 & 0.0 & 20.0 & 2000.0 \\
6 & 1 & 10.0 & 60.0 & 1000.0 \\
6 & 2 & 0.0 & 50.0 & 2000.0 \\
\hline
\end{tabular}

Tab. 1 Generator data.

\begin{tabular}{|rcccc|}
\hline Bus $\mathrm{i}$ & $\begin{array}{c}\mathrm{L}_{\mathrm{i}} \\
(\mathrm{MW})\end{array}$ & $\begin{array}{c}\mathrm{L}_{\mathrm{i}} \\
(\mathrm{MW})\end{array}$ & $\begin{array}{c}\mathrm{L}_{\mathrm{i}} 3 \\
(\mathrm{MW})\end{array}$ & $\begin{array}{c}\mathrm{L}_{\mathrm{i} 4} \\
(\mathrm{MW})\end{array}$ \\
2 & 10.0 & 15.0 & 20.0 & 25.0 \\
3 & 100.0 & 110.0 & 120.0 & 130.0 \\
4 & 50.0 & 60.0 & 65.0 & 70.0 \\
5 & 40.0 & 45.0 & 50.0 & 60.0 \\
6 & 10.0 & 15.0 & 20.0 & 25.0 \\
\hline
\end{tabular}

Tab. 2 Load trapezoidal possibility distributions.

\begin{tabular}{|c|c|c|c|c|}
\hline $\begin{array}{l}\text { branch } \\
\text { numbe }\end{array}$ & $\begin{array}{l}\text { sending } \\
\text { bus }\end{array}$ & $\begin{array}{c}\text { receiving } \\
\text { bus }\end{array}$ & $\begin{array}{c}\mathrm{X} \\
(\mathrm{pu})\end{array}$ & $\begin{array}{l}P_{\text {ijmax }} \\
(\mathrm{MW})\end{array}$ \\
\hline 1 & 1 & 2 & 0.0057 & 80.0 \\
\hline 2 & 1 & 3 & 0.0057 & 112.0 \\
\hline 3 & 2 & 3 & 0.0084 & 100.0 \\
\hline 4 & 3 & 4 & 0.0164 & 100.0 \\
\hline 5 & 4 & 5 & 0.0078 & 80.0 \\
\hline 6 & 5 & 6 & 0.0078 & 110.0 \\
\hline
\end{tabular}

Tab. 3 Line and transformer data.

\section{FOPF algorithm calculations}

The model (20) to (22) was used to evaluate the initial power dispatch presented in Tab. 4.

\begin{tabular}{|cccccc|}
\hline generator section & $\begin{array}{c}\mathrm{G}_{\mathrm{ij} 1} \\
(\mathrm{MW})\end{array}$ & $\begin{array}{c}\mathrm{G}_{\mathrm{ij} 2} \\
(\mathrm{MW})\end{array}$ & $\begin{array}{c}\mathrm{G}_{\mathrm{ij} 3} \\
(\mathrm{MW})\end{array}$ & $\begin{array}{c}\mathrm{G}_{\mathrm{ij} 4} \\
(\mathrm{MW})\end{array}$ \\
1 & 1 & 40.0 & 50.0 & 50.0 & 50.0 \\
1 & 2 & 0.0 & 0.0 & 0.0 & 20.0 \\
1 & 3 & 0.0 & 0.0 & 0.0 & 0.0 \\
2 & 1 & 60.0 & 60.0 & 60.0 & 60.0 \\
2 & 2 & 50.0 & 50.0 & 50.0 & 50.0 \\
2 & 3 & 0.0 & 20.0 & 20.0 & 20.0 \\
6 & 1 & 60.0 & 60.0 & 60.0 & 60.0 \\
6 & 2 & 0.0 & 5.0 & 35.0 & 50.0 \\
\hline
\end{tabular}

Tab. 4 Generator initial trapezoidal possibility distributions. by:

The generated active powers are, thus, given

$$
\begin{aligned}
& \mathrm{G}_{1}<=>(40.0 ; 50.0 ; 50.0 ; 70.0) \mathrm{MW} \\
& \mathrm{G}_{2}<=>(110.0 ; 130.0 ; 130.0 ; 130.0) \mathrm{MW} \\
& \mathrm{G}_{6}<=>(60.0 ; 65.0 ; 95.0 ; 110.0) \mathrm{MW}
\end{aligned}
$$

These generation possibility distributions will now be used to evaluate the power flow possibility distributions using the DC fuzzy power flow model. The corresponding trapezoidal distributions are given in tab. 5.

\begin{tabular}{|ccccc|}
\hline $\begin{array}{c}\text { branch } \\
\text { number }\end{array}$ & $\begin{array}{c}\mathrm{P}_{\mathrm{I} 1} \\
(\mathrm{MW})\end{array}$ & $\begin{array}{c}\mathrm{P}_{\mathrm{I} 2} \\
(\mathrm{MW})\end{array}$ & $\begin{array}{c}\mathrm{P}_{\mathrm{I3}} \\
(\mathrm{MW})\end{array}$ & $\begin{array}{c}\mathrm{P}_{\mathrm{I}} \\
(\mathrm{MW})\end{array}$ \\
2 & -59.6 & -43.0 & -23.6 & -4.2 \\
3 & 29.6 & 63.0 & 103.6 & 135.8 \\
4 & 50.4 & 70.5 & 87.8 & 99.3 \\
5 & -1.0 & 25.0 & 70.0 & 95.0 \\
6 & -60.0 & -35.0 & 5.0 & 25.0 \\
\hline
\end{tabular}

Tab. 5. Power flow trapezoidal possibility distributions.

According to these results, the maximum limit of line 2 power flow was violated. The related $\alpha$ level is 0.7326 . This violation will now be canceled by solving the incremental linear model described by (23), (25), (26), (27), (29) and (30). It must also be said that each generation will now be described by 6 variables as an $\alpha$-cut is now considered. The solution of this incremental linear model produces deviations that will be superimposed on the initial generation 
possibility distributions. However, a solution that does not violate any generator limit is only identified after incorporating in the incremental model 9 constraints related to minimum or maximum generator limits. After performing this iterative process it is possible to evaluate the generations referred to in tab. 6.

\begin{tabular}{|c|c|c|c|c|c|c|c|}
\hline \multicolumn{3}{|c|}{ gen. section $\mathrm{G}_{\mathrm{i}}$} & $\mathrm{G}_{\mathrm{i} 2}$ & $\mathrm{G}_{\mathrm{i} 3}$ & $\overline{\mathrm{G}_{\mathrm{i}}}$ & $\mathrm{G}_{\mathrm{i} 5}$ & $\mathrm{G}_{\mathrm{i} 6}$ \\
\hline & & (MW & (MW & (MW & $(\mathrm{MW})$ & $(\mathrm{MW})$ & $(\mathrm{MW})$ \\
\hline 1 & 1 & 6.6 & 28.6 & 41.6 & 50.0 & 50.0 & 50.0 \\
\hline 1 & 2 & 0.0 & 0.0 & 0.0 & 0.0 & 0.0 & 20.0 \\
\hline 1 & 3 & 0.0 & 0.0 & 0.0 & 0.0 & 0.0 & 0.0 \\
\hline 2 & 1 & 60.0 & 60.0 & 60.0 & 60.0 & 60.0 & 60.0 \\
\hline 2 & 2 & 50.0 & 50.0 & 50.0 & 50.0 & 50.0 & 50.0 \\
\hline 2 & 3 & 0.0 & 0.0 & 0.0 & 20.0 & 20.0 & 20.0 \\
\hline 6 & 1 & 60.0 & 60.0 & 60.0 & 60.0 & 60.0 & 60.0 \\
\hline 6 & 2 & 33.4 & 33.4 & 33.4 & 35.0 & 44.4 & 50.0 \\
\hline
\end{tabular}

Tab. 6. New generation non trapezoidal possibility distributions.

The new generated active power possibility distributions are, thus, given by:

$$
\begin{aligned}
& \mathrm{G}_{1}<=>(6.6 ; 28.6 ; 41.6 ; 50.0 ; 50.0 ; 70.0) \mathrm{MW} \\
& \mathrm{G}_{2}<=>(110.0 ; 10.0 ; 110.0 ; 130.0 ; 130.0 ; 130.0) \mathrm{MW} \\
& \mathrm{G}_{6} \ll=>(93.4 ; 93.4 ; 93.4 ; 95.0 ; 104.4 ; 110.0) \mathrm{MW}
\end{aligned}
$$

The possibility distributions of $\mathrm{G}_{1}$ and $\mathrm{G}_{6}$ are presented in Fig. 7. It should be noted that these distributions have breaking points at level 0.7326 due to the related $\alpha$-cut.

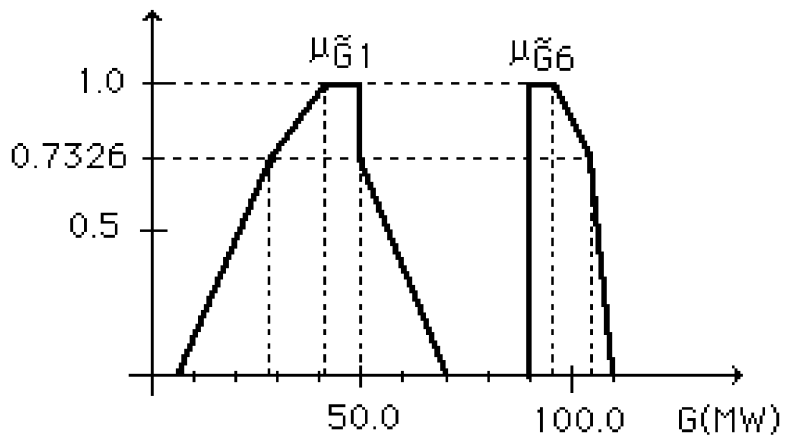

Fig. 7. Generation $\mathrm{G}_{1}$ and $\mathrm{G}_{6}$ possibility distributions.

Afterwards, the DC fuzzy power flow model was used to evaluate the power flow possibility distributions (tab. 7). The branch 4 and 6 power flow possibility distributions are presented in Fig. 8. The transformer power flow possibility distribution is specially interesting as a reversion of the power flow, for the specified uncertainty conditions, can be

\begin{tabular}{|c|c|c|c|c|c|c|}
\hline $\begin{array}{l}\text { branch } \\
\text { (MW) }\end{array}$ & $\begin{array}{l}\mathrm{Pl}_{1} \\
(\mathrm{MW})\end{array}$ & $\begin{array}{r}\mathrm{P}_{\mathrm{I} 2} \\
\quad(\mathrm{M}\end{array}$ & ${ }^{2} \mathrm{P}_{13}$ & $W)^{P_{14}}$ & $\begin{array}{l}\mathrm{P}_{15} \\
N)\end{array}$ & $\begin{array}{c}\mathrm{P}_{16} \\
\mathrm{MW})\end{array}$ \\
\hline 1 & -59.6 & -49.0 & -43.0 & -17.6 & -14.3 & 5.4 \\
\hline 2 & 29.5 & 50.3 & 63.0 & 89.2 & 95.3 & 112.0 \\
\hline 3 & 50.4 & 59.4 & 64.8 & 79.7 & 82.3 & 89.7 \\
\hline 4 & -10.0 & 10.3 & 25.0 & 41.6 & 47.0 & 61.6 \\
\hline 5 & -60.0 & -47.0 & -35.0 & -23.4 & -19.4 & -8.4 \\
\hline 6 & 100.0 & -90.7 & -80.0 & -73.4 & -72.0 & -68.4 \\
\hline
\end{tabular}
detected. This could have, for instance, implications in plant design and protection project.
Tab. 7 New power flow non trapezoidal possibility distributions.

The new power flow distributions don't violate any limit and therefore this solution is considered optimal. In this case, the system has a solution for every future scenario of loads underlying the assumed uncertainty. Therefore, the system exposure index is 0.0 , and the corresponding robustness index is 1.0.

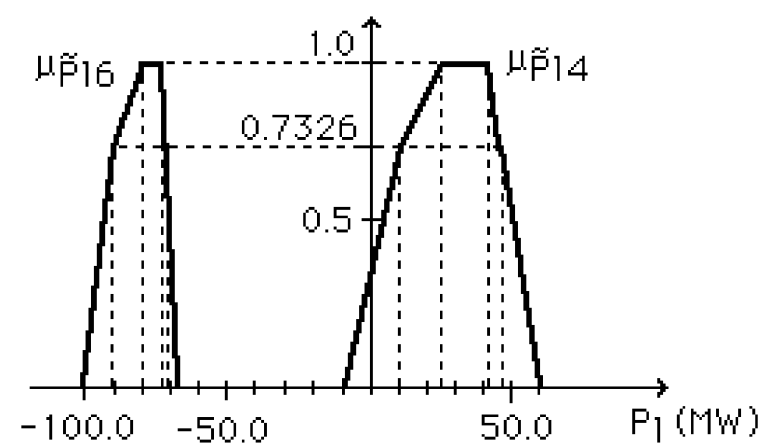

Fig. 8. Transf. 4 and branch 6 power flow possibility distributions.

\section{Hedging strategy}

Let us now admit that the power flow limits of lines 3 and 6 were originally reduced to 70.0 and 80.0 MW, respectively. The incremental FOPF model will now reveal that no generation possibility distributions are able to meet the load uncertainty. Thus, it is possible to evaluate the exposure and robustness index performing the bisection iterative process already described. This process would then lead us to:

$$
\begin{array}{lr}
\text { - exposure index } & \alpha=0.455 \\
\text { - robustness index - } & 1-\alpha=0.545
\end{array}
$$

Finally, an improvement in the robustness index of $20 \%$ is looked for setting an $\alpha$ target of $\alpha^{\prime}=$ 0.364 . By performing an incremental study for a level just below $\alpha=0.455$ it is possible to identify the constraint responsible for the non existence of solutions for $\alpha^{\prime} \in[0, \alpha[$. In this case one concludes that line 6 power flow inferior limit has to be increased to improve the robustness index. The new value of line 6 power flow inferior limit is evaluated by performing a parametric study involving the 4 constraints related to the violation of this line inferior limit. In this case, it is sufficient to change this limit from 80.0 to $84.0 \mathrm{MW}$ to guarantee $\alpha^{\prime}=0.364$. 


\section{CONCLUSIONS}

Dealing with forecasting (specially about the future...) is extremely complex and a need has been always felt for tools to work within a frame of uncertainties. It is now recognized that probabilistic approaches cannot model every kind of uncertainty, and that those having a qualitative nature find in the fuzzy set theory an adequate treatment. With the work described in this paper, we have shown that uncertainties in loads or generations (not of probabilistic type) can be incorporated into power system models so as to give a better (while uncertain) image of system behavior.

In fact, from a DC fuzzy power flow model one can derive possibility distributions of branch power flows from known uncertainty in injected powers. This analysis will always act as a constraint in dispatch decisions, driven by an economic objective. Therefore, the uncertainty in these decisions depends not only on load imprecision but also on eventual corrective measures to insure safe system operation. This global uncertainty, by itself, would lead to a large set of possible decisions; however, an ordering can be imposed in this set in such a way that, in some sense, operation cost and its uncertainty are both minimized.

It can be shown (and not surprisingly) that the optimal fuzzy solutions differ in many cases from those that would have been calculated if only the central values of the possibility distributions were taken. In fact, this seems to be a more realistic system operation forecasting modelling than any deterministic studies.

As a consequence of dealing with fuzzy events, a whole set of load scenarios is analyzed at one time, discarding the need for expensive simulation studies. A direct result of this is having measures of system robustness and exposure to uncertain future scenarios available, and being able to easily identify the critical network elements. Hedging policies can also be investigated, either by setting as target a system robustness value or by analyzing the effect of reinforcements at critical places on the exposure index.

Although several aspects of system behavior are not yet dealt with in this paper, it seems a promising research area to combine the fuzzy approach with probabilistic modelling of, for instance, forced outages of system components. Theoretical tools for such combined analysis of probabilistic and fuzzy numbers are available.

\section{REFERENCES}

[1] V. Miranda, M. A. Matos, "Distribution System Planning with

Fuzzy Models and Techniques", Proceedings of CIRED 1989, Brighton, August 1989.

[2] V. Miranda, M. A. Matos, J. T. Saraiva, "Fuzzy Load Flow - New
Algorithms Incorporating Uncertain Generation and Load Representation", 10th PSCC, Graz, August 1990; in Proceedings of the 10th PSCC, Butterworths, London, 1990.

[3] L. A. Zadeh, "Fuzzy Sets", Information and Control, pp. 338-

353, August 1965.

[4] L. A. Zadeh, "Fuzzy Sets as a Basis for a Theory of Possibility",

International Journal of Fuzzy Sets and Systems, vol. 1, pp. 3-28, January 1978.

[5] D. Dubois, H. Prade, Theorie des Possibilités - Application

à la representation des connaissances en informatique, $2^{\mathrm{a}}$ Edition, Masson, Paris, 1987.

[6] H.-J. Zimmermann, Fuzzy Set Theory - and Its Applications,

Kluwer-Nijhoff Publishing, Boston, 1985.

[7] C. Freksa, "Linguistic Description of Human Judgement in

Expert Systems and in the Soft Sciences", Approximate Reasoning and Decision Control, M. M. Gupta \& E. Sanchez Edt., North Holland, Amsterdam, 1982.

[8] V. Miranda, M. Matos, "A Holistic Approach in Multicriteria

Decision Aid", Improving Decision Making in Organizations, A. G. Lockett Edt., Lecture Notes in Economics and Mathematical Sciences, Springer Verlag, Amsterdam, 1989.

[9] A. Kaufmann, M. M. Gupta, Fuzzy Mathematical Models in

Engineering and Management Science, North Holland, Amsterdam, 1988.

[10] B. Stott, J. L. Marinho, "Linear Programming for Power-

System Network Security Applications", IEEE Transactions on Power Apparatus and Systems, vol. PAS-98, pp. 837-848, May/June 1979.

[11] H. M. Merril, A. J. Wood, "Risk and Uncertainty in Power

System Planning", presented at the 10th PSCC, Graz, Austria, August 1990. 